\title{
On the Impact of Sea State on GNSS-R Polarimetric Observations
}

Mostafa Hoseini ${ }^{1}$, Maximilian Semmling ${ }^{2}$, Erik Rennspiess ${ }^{2}$, Markus Ramatschi², Rüdiger Haas ${ }^{3}$, Joakim Strandberg ${ }^{3}$, Hossein Nahavandchi ${ }^{1}$, and Jens Wickert ${ }^{2,4}$

${ }^{1}$ Department of Civil and Environmental Engineering, Norwegian University of Science and Technology NTNU, Trondheim, Norway

2 Department of Geodesy, German Research Centre for Geosciences GFZ, Potsdam, Germany

${ }^{3}$ Department of Space, Earth and Environment, Chalmers University of Technology, Gothenburg, Sweden ${ }^{4}$ Technische Universität Berlin, Berlin, Germany 
Objective

Analysis of the response of polarimetric GNSS-Reflectometry observations to different sea states 


\section{a Main Dataset}

- Ground-based coastal GNSS-R observations

- Time span of the data: one year, from Jan to Dec 2016

- Sampling interval: 10 second

\section{Ancillary Datasets}

- Water temperature

- Daily estimate of salinity

- Wind speed
To be used in a model to estimate permittivity 


\section{Experiment setup}

- Zenith-looking master antenna for direct signal tracking

- Two side-looking RHCP and LHCP separate antennas to perform polarimetric observations

- About $3 \mathrm{~m}$ above sea level

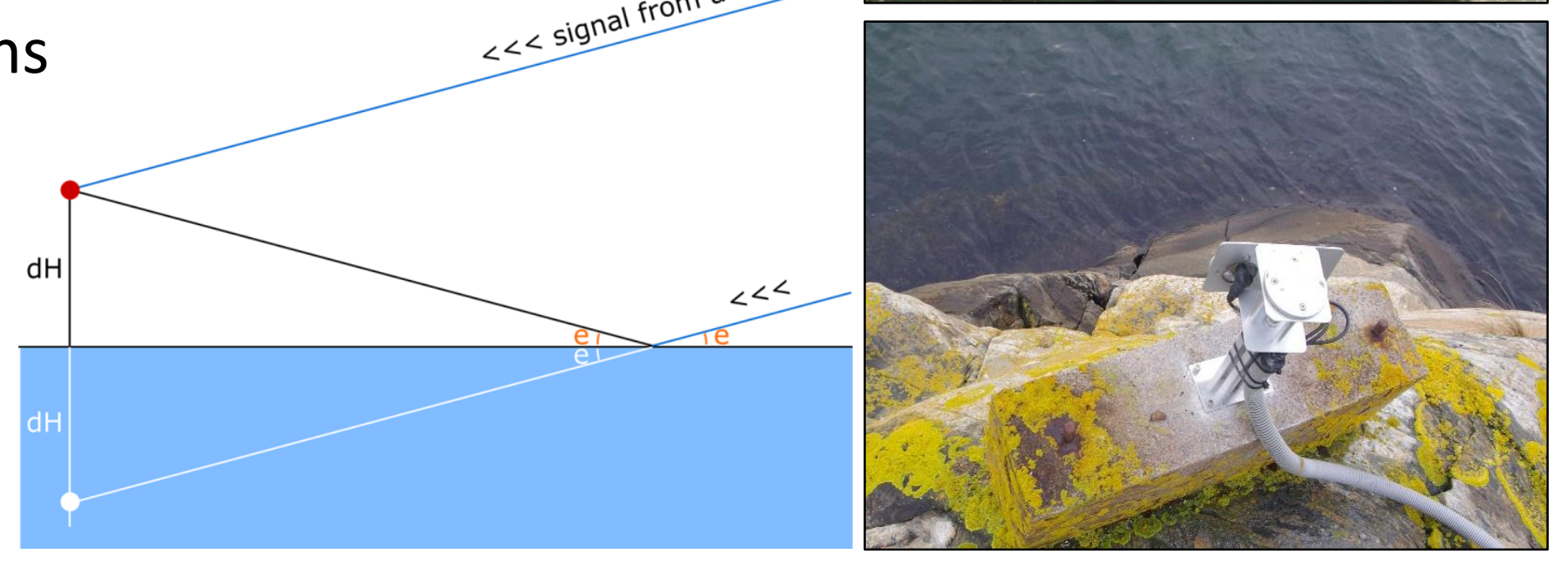




\section{a Method}

- Coherent in-phase and quadrature $(I, Q)$ correlation sums are provided by the receiver - data level 0

- Reflected and direct signal powers from RHCP and LHCP samples are combined in three power ratios - data level 1

- Ratio estimates are inverted to sea surface roughness based on the permittivity of water calculated using ancillary data - data level 2 


\section{Examples of the data level 0 from the receiver

\section{Samples from co-polar link (RHCP antenna)}

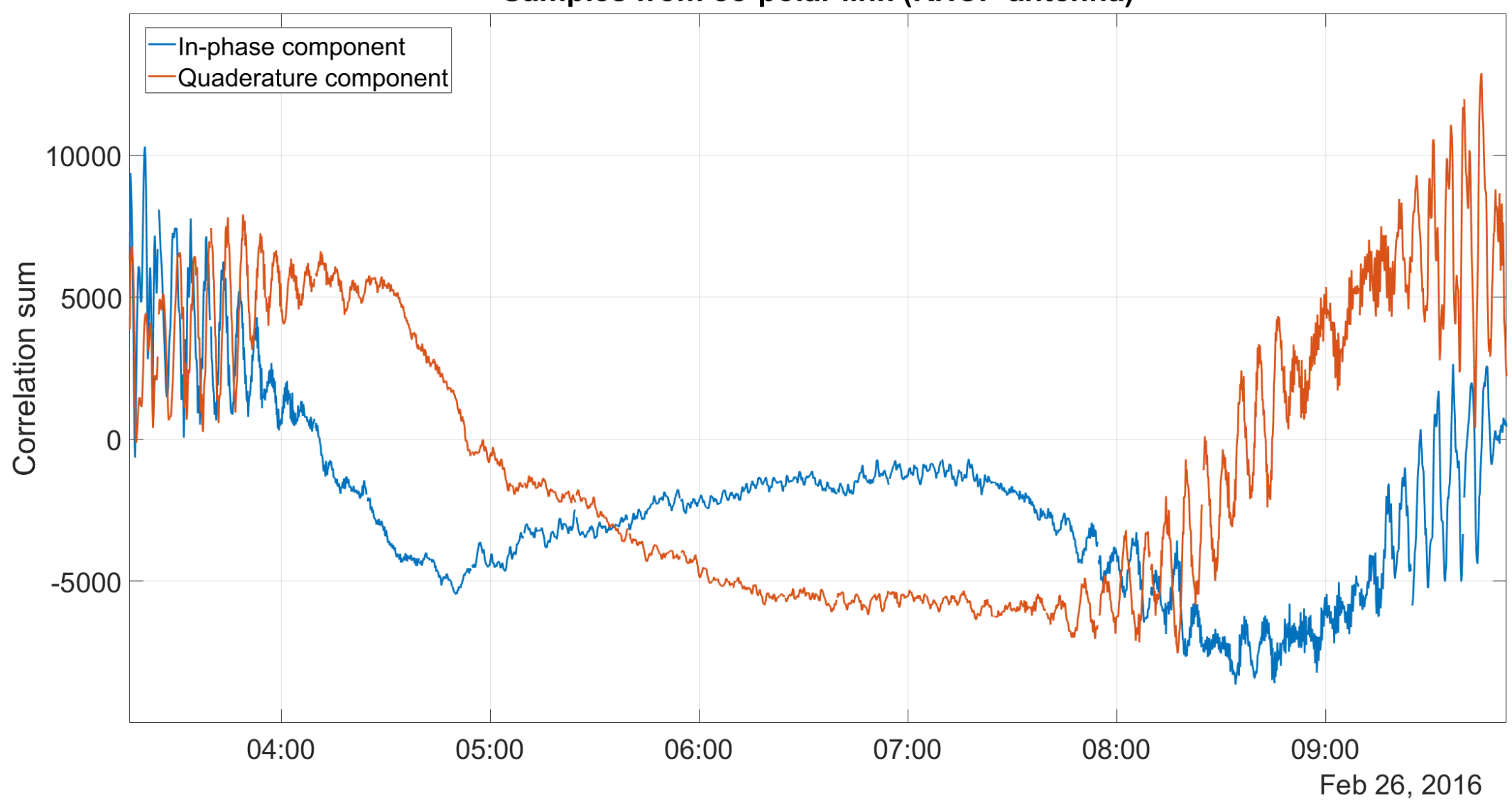




\section{E Examples of the data level 0 from the receiver}

Samples from cross-polar link (LHCP antenna)

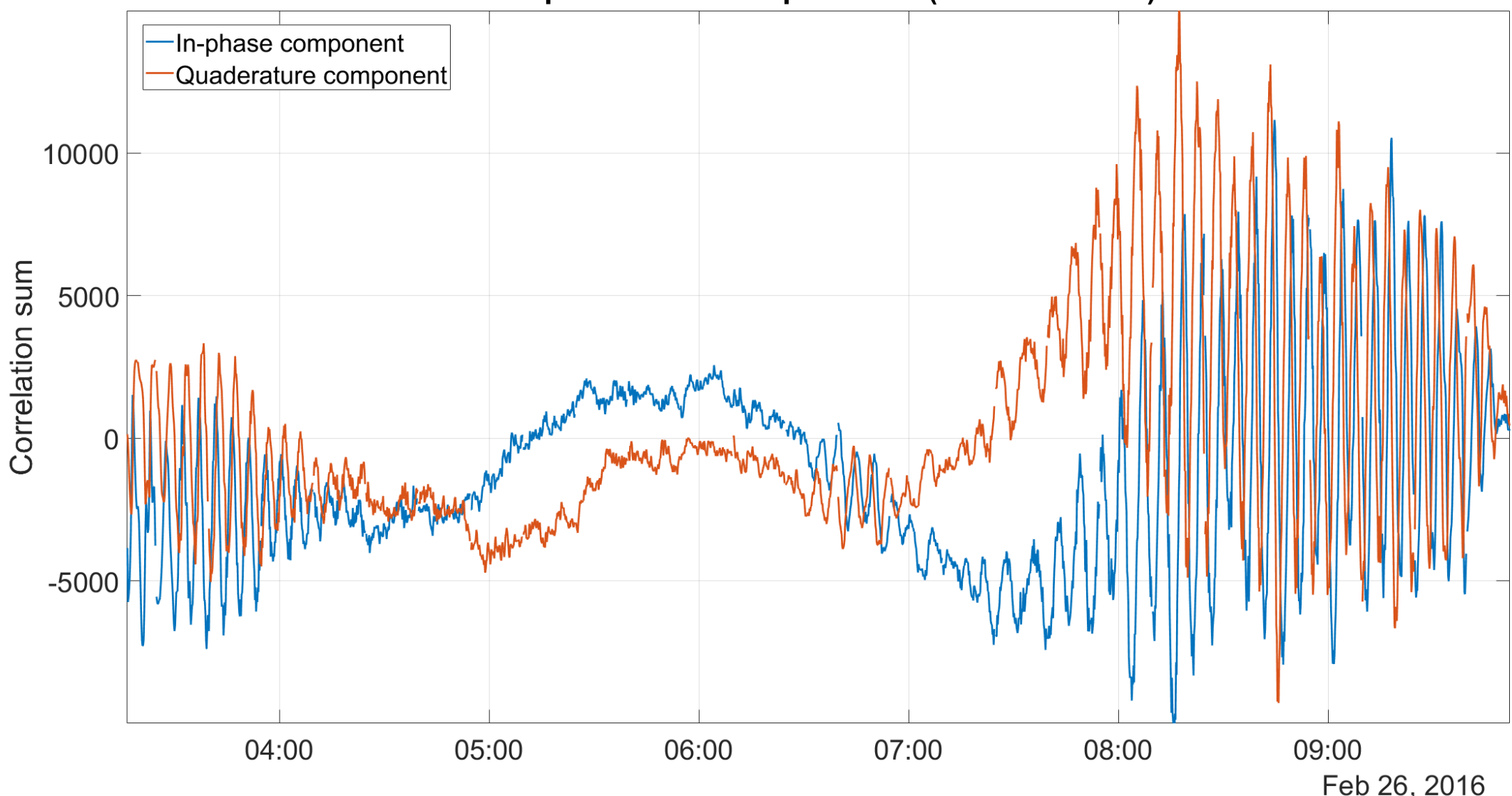




\section{Processing Flow:}

$0.1 \mathrm{~Hz}$ In-phase and quadrature samples from RHCP and LHCP links
Power ratios from the estimates of the direct and reflected signals' power

\section{Roughness}

estimates using a

polarizationindependent model

$$
\left.\begin{array}{l}
P_{21}=\left|R^{x}\right|^{2}\left|S_{2}\right|^{2} \\
P_{31}=\left|R^{c}\right|^{2}\left|S_{3}\right|^{2} \\
P_{23}=\frac{\left|R^{x}\right|^{2}}{\left|R^{c}\right|^{2}}\left|S_{23}\right|^{2}
\end{array}\right\} \begin{gathered}
S(\sigma)=e^{-\frac{1}{2} \frac{(2 \pi)^{2}}{\lambda^{2}} \sigma^{2} \sin ^{2} \theta} \\
\sigma: \text { Standard deviation of } \\
\text { sea surface height }
\end{gathered} \quad \begin{aligned}
& \mathrm{P}_{21}: \text { Cross-polar power ratio, } \\
& P_{31}: \text { co-polar power ratio, } \mathrm{P}_{23}: \text { cross to co polar power ratio }
\end{aligned}
$$




\section{Co-polar vs cross-polar power ratios}

\section{In different wind speeds}




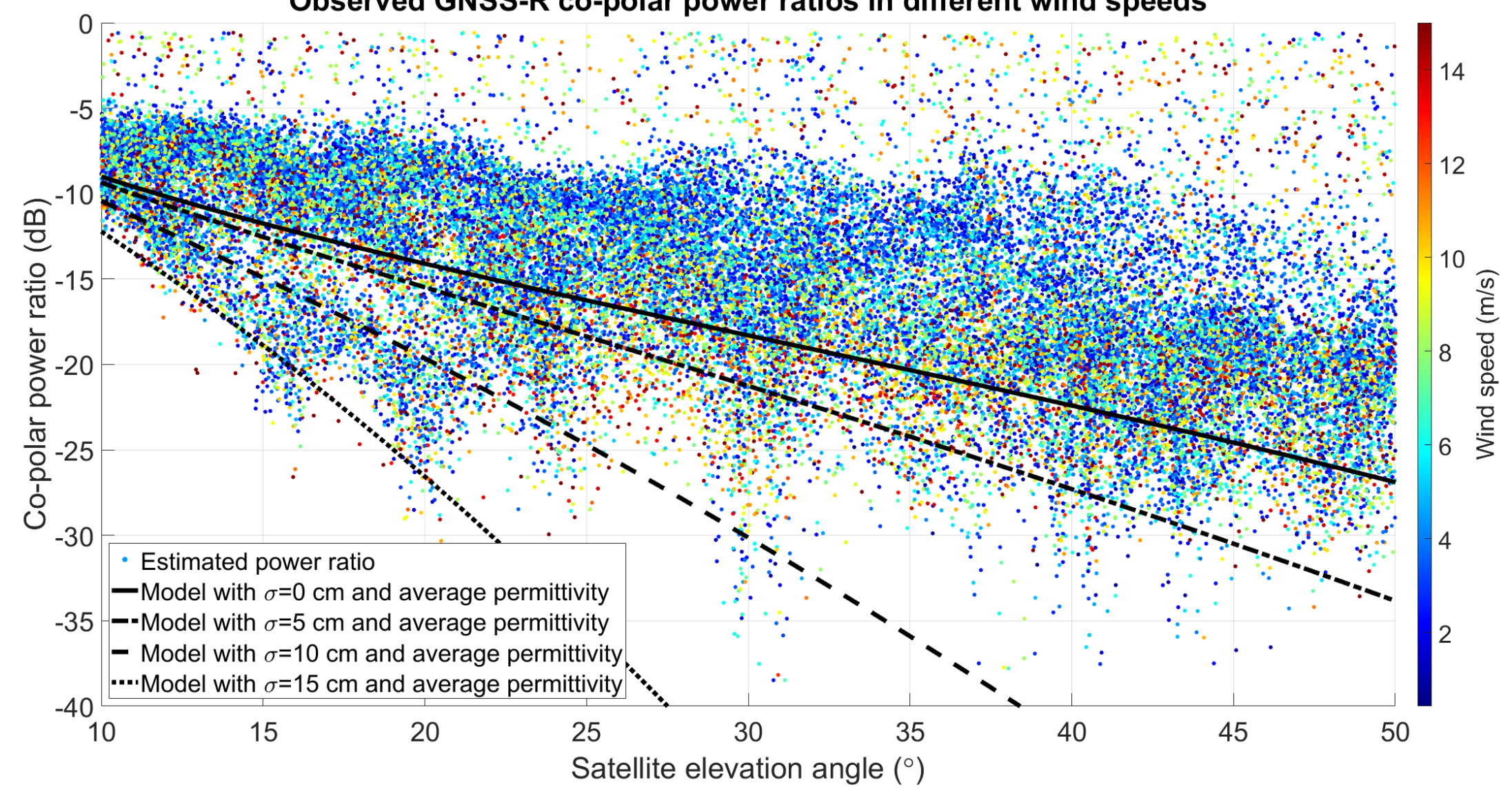




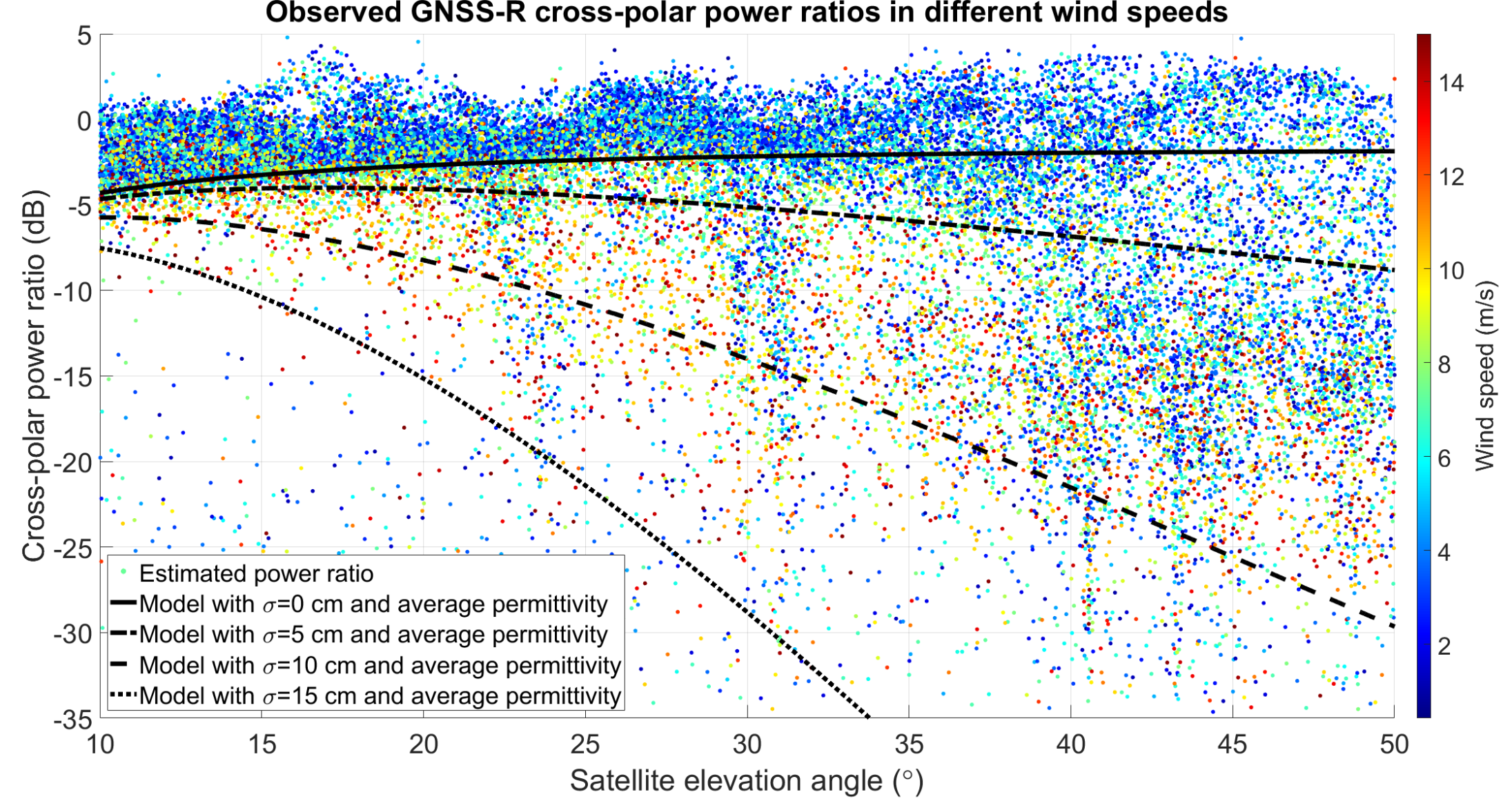




\section{Distribution of power ratios}
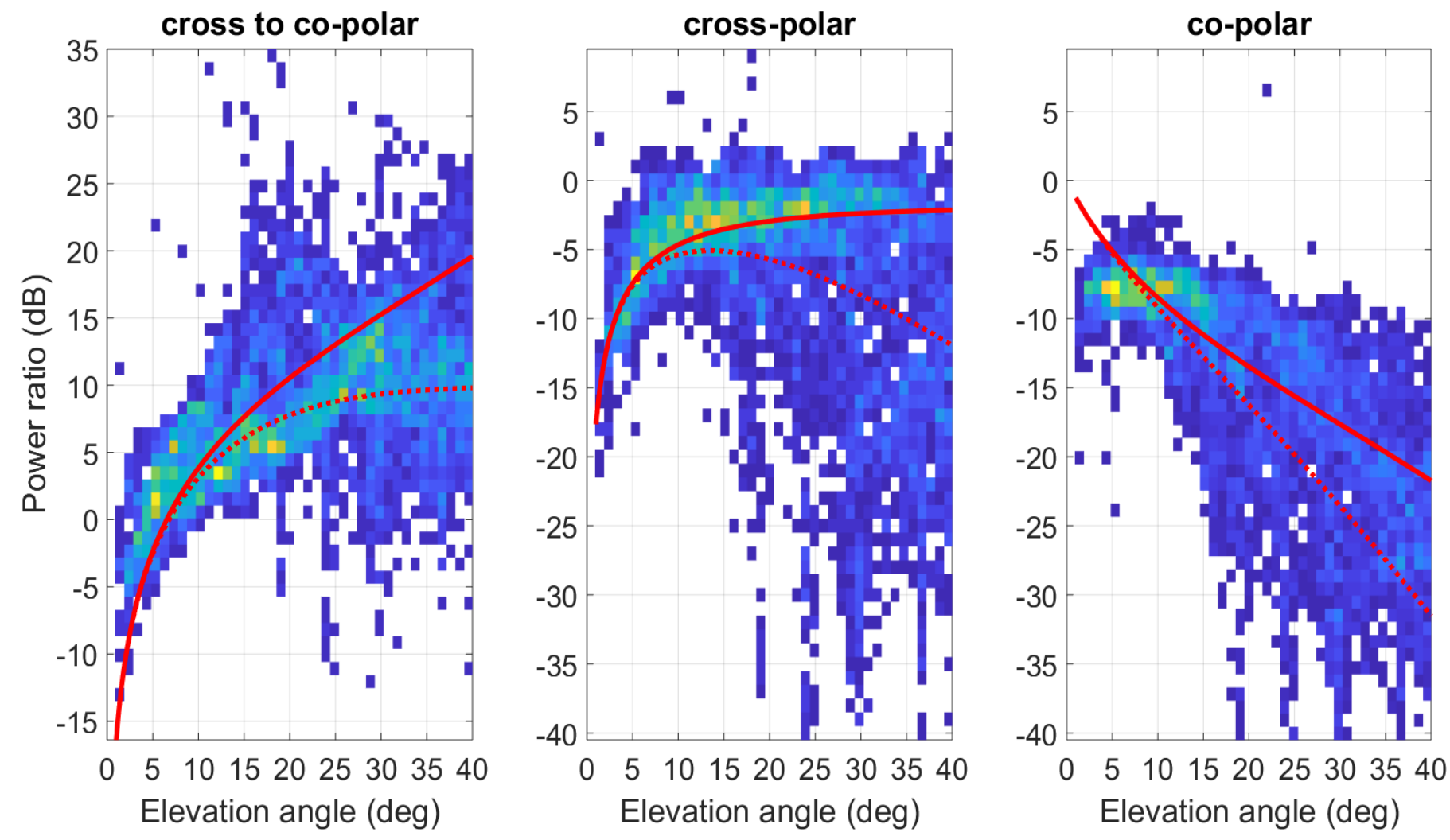


\section{a Roughness estimates vs wind speed}

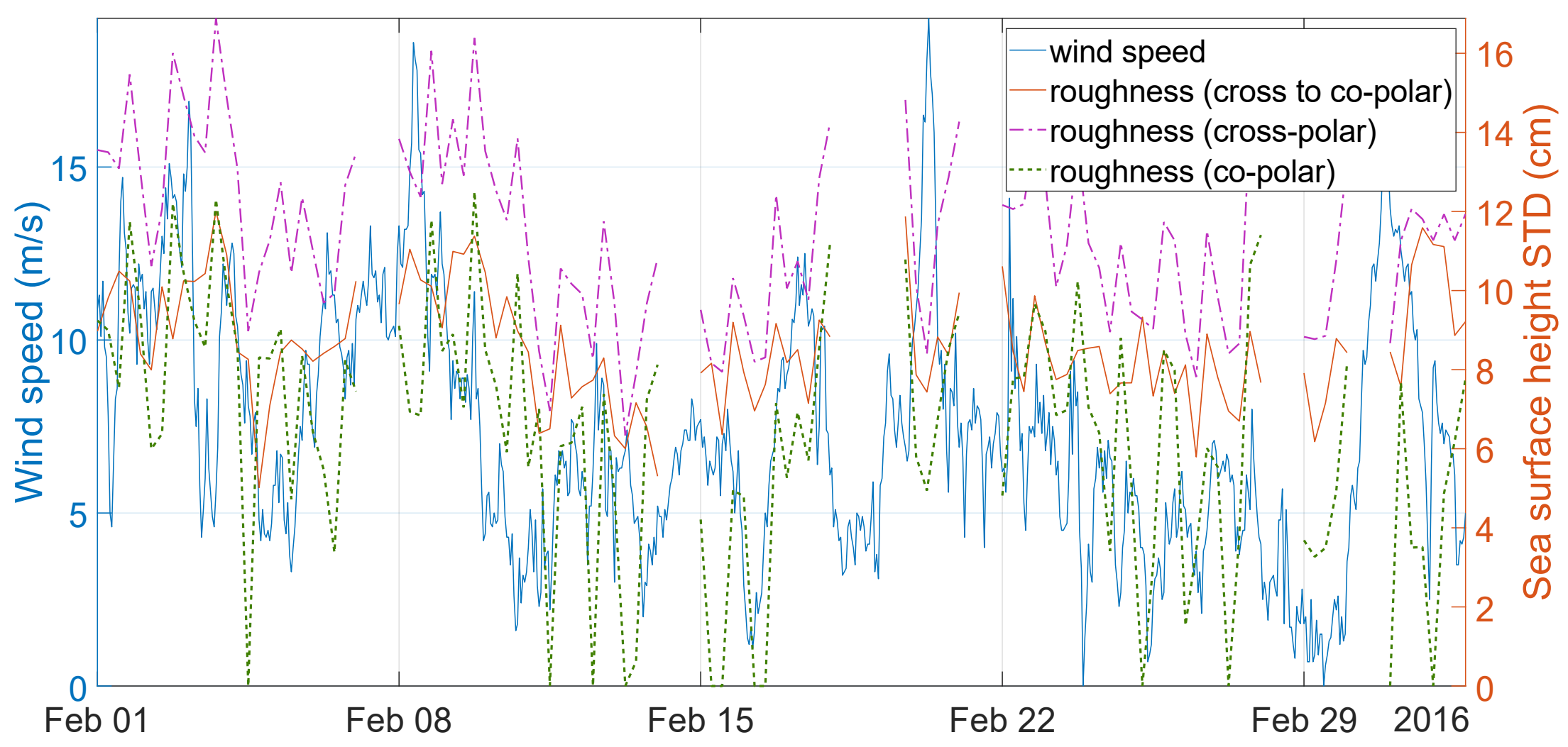


- The effect of different wind speeds as an indicator of sea states on polarimetric observations has been demonstrated using a dataset from a ground-based setup

- Higher sensitivity to the surface roughness is observed for the cross-polar power ratios compared to the co-polar measurements

- The cross-polar power ratios exhibit a pattern in which low wind speeds show higher ratios and high wind speeds show lower ratios

- The model needs enhancement to better describe roughness effect particularly for the co-polar ratios in low elevation angles 


\section{Acknowledgement}

- The Onsala Space Observatory is acknowledged for hosting the respective ground-based measurements and providing in-situ observations of water temperature.

- The Swedish Meteorological and Hydrological Institute is acknowledged for the wind speed and salinity data. 


\section{References}

[1] F. G. Nievinski and K. M. Larson, "Forward modeling of GPS multipath for nearsurface reflectometry and positioning applications," GPS solutions, vol. 18, no. 2, pp. 309-322, 2014.

[2] A. M. Semmling et al., "Sea-Ice Concentration Derived From GNSS Reflection Measurements in Fram Strait," IEEE Transactions on Geoscience and Remote Sensing, 2019.

[3] A. Helm, G. Beyerle, R. Stosius, O. Montenbruck, S. Yudanov, and M. Rothacher, "The GNSS occultation, reflectometry, and scatterometry space receiver GORS: Current status and future plans within GITEWS," in Proceedings of the ESA 1st Colloquium, Scientific and Fundamental Aspects of the Galileo Programme, Toulouse, France, 2007, pp. 1-4.

[4] W. Liu et al., "Coastal sea-level measurements based on GNSS-R phase altimetry: A case study at the Onsala Space Observatory, Sweden," IEEE Transactions on Geoscience and Remote Sensing, vol. 55, no. 10, pp. 5625-5636, 2017. 